\title{
« Remarques sur le discours d'Hannibal à Scipion »
}

Notes et édition du texte Jean-Yves Vialleton

Jean-Pierre de Crousaz

Jean-Yves Vialleton (éd.)

\section{(2) OpenEdition}

Journals

Édition électronique

URL : http://journals.openedition.org/rhetorique/194

DOI : 10.4000/rhetorique.194

ISSN : 2270-6909

Éditeur

UGA Éditions/Université Grenoble Alpes

Édition imprimée

ISBN : 978-2-84310-263-9

\section{Référence électronique}

Jean-Pierre de Crousaz, " « Remarques sur le discours d'Hannibal à Scipion » », Exercices de rhétorique [En ligne], 2 | 2013, mis en ligne le 19 novembre 2013, consulté le 12 septembre 2020. URL : http:// journals.openedition.org/rhetorique/194; DOI : https://doi.org/10.4000/rhetorique.194

Ce document a été généré automatiquement le 12 septembre 2020

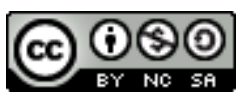

Les contenus de la revue Exercices de rhétorique sont mis à disposition selon les termes de la Licence Creative Commons Attribution - Pas d'Utilisation Commerciale - Partage dans les Mêmes Conditions 4.0 International. 


\title{
« Remarques sur le discours d'Hannibal à Scipion »
}

\author{
Notes et édition du texte Jean-Yves Vialleton
}

Jean-Pierre de Crousaz

Jean-Yves Vialleton (éd.)

1 Pour bien juger d'un discours, il faut d'abord se former une juste idée du sujet sur lequel il roule, et de toutes ses circonstances. Il faut ensuite se rendre attentif au caractère de celui qui le prononce, et au caractère de celui à qui il s'adresse.

2 Pour persuader il faut alléguer des raisons, et il faut que l'ordre dans lequel on les range, et le choix des expressions dans lesquelles on les énonce, se trouvent propres à en faire sentir la force; Cela est nécessaire; mais cela ne suffit pas. Il faut se faire écouter, il faut s'emparer de l'attention et du cœur ${ }^{1}$ de ceux à qui l'on s'adresse, il en faut étudier le caractère et la [27] situation présente, afin de s'y régler. Mais il importe outre cela qu'un Orateur s'attire l'estime et la considération de son Auditeur : Il doit se connaître lui-même, et se soutenir ${ }^{2}$ dans le caractère sous lequel il lui est avantageux de se présenter. Il lui est permis de faire connaître ${ }^{3}$ la grandeur de ses sentiments pourvu qu'il n'aille point jusqu'à se faire soupçonner de fiertét. Il peut utilement faire sentir à quel point son sort est digne de compassion, pourvu que par ses plaintes, et par l'aveu de sa misère, il ne s'expose pas à se voir méprisé5.

Hannibal après une longue suite de victoires, essuie les plus cruels revers ${ }^{6}$. Après avoir été sur le point d'entrer triomphant dans Rome, il se voit forcé d'abandonner l'Italie, pour courir au secours de Carthage prête à tomber sous les efforts de Scipion. Il se voit l'Auteur d'une Guerre qui a mal réussi, et il est cause qu'on l'a continuée, au lieu de profiter de ses heureux commencements, et de la terminer par une paix avantageuse : Il sait qu'il a dans sa Patrie un grand nombre d'ennemis qui, jaloux de sa gloire, seraient ravis de pouvoir l'accabler. Il voit qu'il a à combattre des Légions accoutumées à vaincre, et pleines de confiance en un Chef également habile et heureux: Il craint qu'une [28] longue suite de malheurs n'ait fait perdre à ses troupes celle qu'elles avaient en lui. Il considère que l'armée de Scipion ne peut avoir d'espérance qu'en la victoire, au lieu que ses Soldats ont les murs de Carthage pour retraite, et savent que la 
fuite les peut garantir de la mort. Toutes ces raisons lui font souhaiter la paix. Il sait que Carthage la demande, et qu'on n'y est pas d'humeur à continuer la guerre. Il voudrait seulement que sa présence contribuât à la terminer un peu plus avantageusement.

Hannibal a trop d'esprit et trop d'expérience pour douter que Scipion ne connaisse et la faiblesse des Carthaginois, et l'embarras de leur Général. Mais il rassemble tout ce qu'il a de génie ${ }^{7}$ pour se saisir de quelque endroit, par où cet Illustre Romain puisse se laisser gagner. Il connaît en Scipion de la grandeur d'âme, de la politesse ${ }^{8}$, et de la piété 9 . Il met habilement en œuvre tout ce qui est capable de l'ébranler par ces trois endroits. Hannibal ne fait point le fier il ne dissimule point son inclination pour la paix; il l'aurait en vain déguisée. Il cherche à gagner la confiance de Scipion, il s'énonce avec franchise, ses expressions semblent partir du cœur : Vous diriez que ce sont les intérêts de Scipion [29] même qui le font parler ${ }^{10}$. Tel est le but d'Hannibal, telles sont les dispositions de son âme. Voyons avec quelle adresse il va à son but.

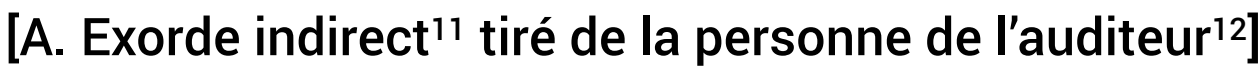

\section{[Aa]}

[Tite-Live, XXX, 30, § 3] Si hoc ita fato datum erat, ut qui primus bellum intuli populo Romano, quique toties prope in manibus uictoriam habui, is ultro ad pacem petendam uenirem, laetor te mihi potissimum datum a quo peterem.

$\mathrm{Si}$, par un ordre irrévocable du Destin après avoir été le premier Mobile de cette seconde guerre des Carthaginois avec les Romains, et m'être vu si souvent sur le point de la terminer par mes Victoires, il était arrêté que ce fût encore à moi à venir demander la paix ; dans cette extrémité où je me vois réduit, il me reste un sujet de joie, c'est que c'est à vous ${ }^{13}$ à qui le même Destin veut encore que je m'adresse, et qu'il est surtout en votre ${ }^{14}$ pouvoir de me faire obtenir ce que je demande.

5 Il ne se pouvait que Scipion ne fût très sensible à la gloire de voir Hannibal, qui avait si souvent fait trembler les Romains, venir lui demander la paix, à la vue des remparts de Carthage. Hannibal, qui ne cherche qu'à gagner le cœur de Scipion, n'a garde de le détourner d'une réflexion si flatteuse ; C'est par là qu'il commence son discours. Il sait qu'il faut plaire pour être écouté et pour persuader. Mais en [30] reconnaissant les avantages de Scipion et en le félicitant de sa gloire, il ne se méprise point, et ne se trouve point méprisable, quoiqu'il se voie réduit à faire le personnage de suppliant ${ }^{15}$. C'est le sort dit-il qui en a décidé.

6 SI HOC ITA FATO DATUM ERAT ${ }^{16}$ : Rejeter sur le Destin ses mauvais succès est une pensée commune ; Mais par là même elle sert mieux Hannibal ; Il commence par une réflexion des plus communes; un tel Exorde n'a rien de suspect, il n'est point recherché, il est tout propre à faire regarder Hannibal comme un homme qui ne pense point à surprendre, et qui n'a point étudié un discours dans ce dessein.

7 D'un côté Scipion qui dès sa jeunesse, s'était rendu respectable par sa piété, savait qu'il faut respecter les ordres du Destin, et qu'il y a de l'inhumanité à insulter à ceux qu'il persécute. D'un autre, Hannibal paraît toujours grand et digne d'une meilleure destinée, par la manière dont il pense dans la mauvaise ; Il ne murmure point, et il ne porte point envie à ceux qui sont plus heureux : Il voit toute la gloire de Scipion, il la lui met devant les yeux ${ }^{17}$, et puisqu'il devait être abaissé, il se console que ce soit par l'élévation d'un tel rival. 
8 Un exorde ${ }^{18}$ de cette nature n'aurait [31] servi qu'à rendre toujours plus intraitable un homme féroce; mais il ne se pouvait que Scipion, dont l'Âme était véritablement grande, et qui avait une grande piété et une grande politesse, ne fût sensible à ces douceurs, et ne souhaitât de mériter toute cette estime et toute cette affection, dont Hannibal lui donnait de si fortes assurances.

Outre cela Hannibal va adroitement à son principal but, et il y va d'autant plus sûrement, que sa marche est plus couverte ${ }^{19}$. Il comprend que s'il peut une fois amener Scipion à réfléchir bien sérieusement sur les bizarreries du sort, et sur l'incertitude de la destinée, il en obtiendra aisément la préférence d'une paix sûre à une victoire douteuse $\mathrm{e}^{20}$. Il sème dès son exorde, des idées propres à faire naître ces réflexions : Il les proposera en termes exprès dans la suite, il $\mathrm{y}$ appuiera ${ }^{21}$; mais dès le commencement il laisse déjà échapper de quoi y préparer son grand Auditeur. En félicitant Scipion, pour gagner sa confiance par des démonstrations d'amitié et de grandeur d'âme ${ }^{22}$, il fait des oppositions de sa fortune avec celle de ce Général, très propres à faire craindre l'incertitude des événements ${ }^{23}$.

10 Le nom d'Hannibal suffisait pour réveiller toute l'animosité des Romains, à qui il [32] avait tant fait de mal, mais il désarme la vengeance de Scipion, en se présentant désarmé et déjà puni par la destinée, qui l'a réservé à demander la paix ${ }^{24}$.

11 Ses démarches ne sont pourtant point chez lui un pur effet de la nécessité, Ultro dit-il, ueni ad pacem petendam. Je consens à la demander, je m'y porte de moi-même. Ultro ce terme est propre à attendrir. En parlant de ses Victoires il adoucit encore l'expression toties, si souvent, en ajoutant prope in manibus habui, J'ai été sur le point, ou peu s'en est fallu, comme si effectivement il n'avait jamais remporté que des victoires douteuses ${ }^{25}$.

\section{[Ab]}

12 Après cela il continue de faire sentir à Scipion toute la gloire dont il jouissait déjà, pour l'engager à s'en contenter.

[§ 4] Tibi quoque inter multa egregia non in ultimis laudum hoc fuerit, Hannibalem, cui de tot Romanis Ducibus uictoriam Dii dedissent, tibi cessisse; teque huic bello, uestris priusquam nostris cladibus insigni, finem imposuisse.

Pour vous Scipion, dans cette glorieuse suite de Succès, qui vous élèvent au dessus de tous vos Concitoyens, on ne comptera pas pour un des moins brillants, qu'Hannibal à qui les Dieux ont donné la Victoire sur tant de Généraux Romains, ait enfin cédé à votre fortune et à votre valeur, et que par là vous ayez [33] mis fin à une sanglante guerre mémorable par vos défaites, longtemps avant que de l'être par les nôtres.

13 Comme la gloire de Scipion se tire surtout de l'abaissement d'Hannibal, cet habile Orateur ne dissimule pas celle où il s'est vu, dans le dessein où il est de faire mieux sentir la grandeur de sa chute, cui de tot Romanis Ducibus uictoriam, il s'attribue donc un grand nombre de victoires, mais il adoucit encore cette expression en rapportant ces victoires, aux Dieux, cui de tot Romanis Ducibus uictoriam Dii dedissent, les Dieux me l'ont donnée.

14 Par là il engage Scipion à penser qu'Hannibal a aussi été le favori des Dieux, et à réfléchir que ces Dieux refusent la continuation de leurs grâces et de leur protection à ceux qui en abusent; Toutes ces insinuations étaient pleines de force sur un cœur où la piété régnait. 

par le mot de clades. L'idée des défaites affreuses des Romains qui ont précédé leurs victoires, est propre à faire trembler pour l'avenir ; mais de peur que cette idée n'irrite, le correctif vient incontinent, finem imposuisse, mettre fin à cette sanglante guerre.

[34] À intuli bellum, il oppose, uenio ad pacem petendam. À Bello uestris cladibus insigni, il oppose, finem imposuisse. Dans la seconde Antithèse, au premier membre plus fort répond aussi le second plus flatteur. J'ai porté la guerre: Je viens demander la paix. Elle a été mémorable par vos défaites: C'est vous qui la ferez cesser.

La première période est plus simple ; c'est ainsi qu'il faut commencer. La seconde est pompeuse $^{26}$ : La gloire de Scipion élève le style d'Hannibal, il ne dissimule point qu'il croit que ses malheurs portent cette gloire à son comble, par cette idée il va à son but ; Un cœur ambitieux et généreux refuse difficilement son affection à celui qui fournit la plus grande matière de sa gloire ; mais Hannibal, en disant cette vérité, qui va à le louer lui-même, tempère cette louange par sa modestie, non in ultimis laudum, au lieu de dire, in maxima laude. Il ne dit pas ce sera le plus haut point de votre gloire. Il se contente de dire. Ce ne sera pas un des moindres.

\section{[Ac]}

[§5] Hoc quoque ludibrium Fortunae casus ediderit, ut cum Patre tuo Consule ceperim arma, cum eodem primo Romano Imperatore signa contulerim, ad filium eius inermis ad pacem petendam ueniam.

D'autres circonstances fatales serviront encore à faire connaître jusqu'où la Fortune se plaît à se jouer des hommes. Ce fut sous le Consulat de votre Père, que je me mis à la tête de mon armée, Il fut le premier Général à qui je [35] livrai bataille, et c'est son Fils qui me voit désarmé lui demander la paix.

18 À la vue d'Hannibal il n'est pas possible que Scipion ne se souvienne de la défaite de son Père, surtout après ce que ce Général vient de dire de la guerre, Cladibus uestris insigni. Pour prévenir les Sentiments où pouvait jeter cette Idée, Hannibal fait connaître à Scipion qu'il est déjà vengéê ${ }^{27}$. Cepi arma, contuli signa, inermis ad pacem petendam uenio.J'ai pris les armes, j'ai livré bataille; Je viens demander la paix. Il l'avait déjà dit, mais en insérant ce mot d'inermis, désarmé, il le dit avec une nouvelle force ${ }^{28}$.

Cet endroit était délicat, mais il fallait pourtant le toucher ; Hannibal le fait avec toute l'habileté imaginable, en parlant de son humiliation, il n'épargne point les termes, Inermis uenio ; mais en parlant de sa gloire précédente, il choisit les expressions les plus faibles, cepi arma, signa contuli : J'ai pris les armes, j'ai livré bataille. Il ne se représente point comme vainqueur. Ces ménagements se font sentir, et ne peuvent $\left[3^{29}\right]$ être sans effet ; ils vont à faire penser qu'Hannibal se repent.

Il n'y a pas une période où Hannibal ne trouve moyen de présenter à Scipion, sous diverses faces et sous divers prétextes, la prodigieuse variété des événements, dans la guerre, Hoc quoque lubidrium casus ediderit Fortuna. Voici encore un Jeu de la Fortune.

21 Les dernières paroles d'Hannibal donnent l'idée d'un homme qui se repent, et qui se refuse le plaisir de penser à ce que le passé a eu de favorable pour lui : Ce repentir l'amène à des réflexions sérieuses, qui lui font condamner le passé universellement. 


\section{[B. Réflexions sérieuses : opposition de l'équité, des douceurs de la paix et de la sûreté aux incertitudes de la guerre $\left.{ }^{30}\right]$}

$[\mathrm{Ba}]$

[§ 6] Optimum quidem fuerat, eam patribus nostris mentem datam a Diis esse, ut et uos Italiae, et nos Africae imperio contenti essemus; [\$ 7] neque enim ne uobis quidem Sicilia, atque Sardinia satis digna pretia sunt, pro tot classibus, tot exercitibus, tot tamque egregiis amissis ducibus.

À la vérité s'il avait plu aux Dieux de donner à vos Ancêtres, et aux miens, assez de lumière et de modération pour se borner, vous à régner sur l'Italie, et nous à être les Maîtres de l'Afrique, rien ne pouvait arriver ${ }^{31}$ de plus heureux, ni pour les uns ni pour les autres; car enfin il s'en faut beaucoup que la Sicile et la Sardaigne ne soient d'un prix à [37] vous dédommager de tout ce par où vous les avez acquises ${ }^{32}$, de tant d'armées de Terre et de Mer et de tant de Généraux du premier mérite, que vous avez vu périr avant que de vous en voir les possesseurs.

Jusqu'ici la personne même de Scipion est entrée dans tout ce qu'Hannibal vient de dire ; mais il la quitte cette idée [sic] pour passer à des maximes générales; son discours en devient plus grave, et dispose celui qui l'écoute à faire de plus sérieuses réflexions ${ }^{33}$.

Afin que Scipion se trouve plus sensible à cette réflexion sérieuse d'Hannibal, et qu'il y entre avec lui, il parle encore des Dieux, dont il savait que Scipion aimait à consulter la volonté, ut ea mens a Diis immortalibus. Que les Dieux n'ont-ils inspiré cette pensée.

On pouvait lui objecter, les réflexions vous conviennent à vous qui n'avez fait que des pertes, mais pour nous qui avons gagné des Provinces, nous n'avons pas lieu de nous repentir de nos guerres. Hannibal prévient cette objection ${ }^{34}$ en disant, neque enim ne uobis quidem Sardinia atque Sicilia ${ }^{35}$ satis digna pretia sunt, pro tot classibus, tot exercitibus, tot tamque egregiis Ducibus amissis ${ }^{36}$; car enfin la Sicile et la Sardaigne, méritent-elles d'avoir été achetées par tant de pertes. Classes, Exercitus, Duces, tot Duces, Duces [38] egregii, tam egregii, Des Flottes, des Armées, des Généraux, des Généraux d'un mérite si distingué. Chaque terme enchérit sur les précédents ${ }^{37}$.

Il amène Scipion à opposer à l'Idée de l'Italie peuplée, riche, et en possession des fruits d'une longue paix, l'Idée de l'Italie épuisée d'hommes et d'argent, par la défaite de ses armées, et par la perte de ses flottes. Tout cela tend à rendre l'idée de la paix plus aimable que celle de la guerre ${ }^{38}$.

\section{$[\mathrm{Bb}]$}

Il est assez naturel aux hommes de s'évaporer en réflexions sur le passé ; c'est même là une consolation fort ordinaire aux misérables; mais c'est une consolation des plus faibles, et des plus inutiles. Mais ceux qui ont de la prudence au lieu de se borner à des souhaits infructueux, et à regretter le passé sur lequel ils ne peuvent rien, en profitent pour mieux régler l'avenir. Aussi Hannibal, pour parler en homme que l'expérience a solidement instruit, applique incessamment le passé aux circonstances présentes :

Sed praeterita magis reprehendi possunt quam corrigi. [§ 8] Ita aliena appetiuimus, ut de nostris dimicaremus, nec in Italia solum uobis bellum, nobis in Africa esset; sed et uos in portis uestris prope ac moenibus signa, armaque hostium uidistis, et nos ab [39] Carthagine fremitum castrorum Romanorum exaudimus. 
Mais il est beaucoup plus facile de se plaindre du passé, que d'y apporter du remède ${ }^{39}$. Tel a été l'effet de notre ambition qu'en cherchant à nous emparer de ce qui appartenait aux autres, nous avons risqué de perdre ce dont nous étions les légitimes possesseurs : Vous avez eu la guerre en Italie, nous l'avons en Afrique. Il y a plus. Presque à vos portes et depuis vos murailles vous avez vu flotter les drapeaux, et briller les armes de vos ennemis, tout comme aujourd'hui l'on entend à Carthage le bruit de votre Camp.

Des idées du passé Hannibal vient donc au présent, pour le mieux régler. Il le met sous les yeux de Scipion, et toujours fait sentir les inégalités de la fortune, qui se plaît à passer d'un parti à l'autre, et de tout égaler, par des bizarreries mêmes. Sed praeterita magis reprehendi possunt quam corrigi. Il est plus facile de se plaindre du passé, que de le corriger. Par cette maxime pleine de sens il engage Scipion à une attention nouvelle. Ce qu'il ajoute immédiatement ne peut encore que lui plaire; il lui présente une idée agréable aux Romains. Ita aliena appetiuimus, ut nostris dimicaremus. Nous avons porté nos désirs sur le bien des autres ; Nous avons risqué le nôtre.

\section{[Bc]}

Il semble [40] condamner dans ses paroles, les Carthaginois; mais il en ajoute d'instructives qui font voir à Scipion les Romains dans le même cas :

Nec in Italia solum uobis bellum, nobis in Africa est, sed et uos in portis uestris prope ac moenibus signa armaque hostium uidistis, et nos ab Carthagine fremitum castrorum Romanorum exaudiuimus. [§ 9] Quod igitur nos maxime abominaremur, uos ante omnia optaretis, in meliore uestra fortuna de pace agitur.

Vous avez eu la guerre en Italie, nous l'avons en Afrique. Il y a plus. Presque à vos portes et depuis vos murailles, vous avez vu flotter les drapeaux et briller les armes de vos ennemis, tout comme aujourd'hui l'on entend à Carthage le bruit de votre Camp. Ce à quoi nous n'aurions pu penser ci-devant sans en frémir d'horreur, et ce que vous auriez au contraire souhaité comme le plus grand des bonheurs. Il s'agit de faire la paix, quand la Fortune est de votre côté.

Mais cela même pouvait faire craindre aux Romains que les Carthaginois ne tiendraient pas une paix forcée, et à laquelle le mauvais état de leurs affaires les réduisait. Hannibal prévient cette objection, et fait espérer une paix durable.

\section{$[\mathrm{Bd}]$}

Cette espérance était agréable, il répand aussi dans cet endroit de son discours [41] un agrément de paroles ${ }^{40}$; de pace agitur, agimus ii. On parle de paix et c'est nous qui la proposons.

31 Il n'avance pas l'objection pour la résoudre ensuite, car il faut éviter de faire naître des défiances; Mais il lui empêche de se présenter: Il fait voir certitude égale de côté et d'autre par une raison tirée d'un intérêt commun.

\section{[Be]}

L'idée de la Paix doit toujours plaire à un honnête homme, et Hannibal, pour faire mieux comprendre à quel point des sentiments opposés à la paix sont déraisonnables, emploie encore les termes les plus vifs, 
Animo tantum nobis opus est non abhorrente a quietis consiliis.

Pour conclure cette paix nous n'avons besoin les uns et les autres que d'un cœur qui

n'ait pas en horreur des propositions qui tendent à établir notre repos commun ${ }^{41}$.

Rien ne peut rendre infructueuses ces démarches qu'une horreur secrète pour des conseils de paix, et pour des propositions qui tendent au repos des hommes.

\section{[Bf]}

Après avoir fait souvenir Scipion que le sort de deux Républiques est dans leurs mains, idée des plus flatteuses et des plus propre à le gagner, il se présente comme un homme à qui une Raison tranquille laisse voir les choses telles qu'elles sont, et le portrait abrégé qu'il fait de sa vie rend [42] croyable ce qu'il avance ${ }^{42}$.

[§10] Quod ad me attinet, iam aetas senem in patriam reuertentem, unde puer profectus sum, iam secundae, iam aduersae res, ita erudierunt, ut Rationem sequi quam Fortunam malim.

Pour ce qui est de moi qui suis sorti de ma Patrie lorsque j'étais encore dans l'enfance, et qui aujourd'hui y reviens chargé d'année. Le temps et les vicissitudes de la prospérité la plus brillante, et des revers les moins attendus, m'ont rendu assez sage pour préférer désormais constamment ce que la Raison nous conseille, à ce que ${ }^{43}$ la Fortune pourrait nous faire espérer.

\section{[C. Leçon ${ }^{44}$ : intérêt de Scipion]}

\section{[Ca]}

L'exemple d'Hannibal est sans doute propre à gagner une grande âme, mais à cet exemple il s'agissait de joindre encore quelques instructions. C'est ici l'endroit le plus délicat de tout son discours. Naturellement le cœur humain n'aime pas à recevoir des leçons ; Hannibal pour engager Scipion à se rendre attentif aux siennes et à les prendre en bonne part, se présente à lui sous l'idée d'un Père que la tendresse fait parler.

[§ 11] Tuam adolescentiam, et perpetuam felicitatem, ferociora utraque quam quietis opus est consiliis, metuo.

Mais votre ${ }^{45}$ grande Jeunesse, et cette félicité qui ne s'est jamais ${ }^{46}$ démentie, dont

l'effet naturel est d'inspirer de la fierté et de l'éloignement pour des propositions

[43] qui tendent au repos ; Vos avantages mêmes me font craindre pour vous.

Quand on a fait tant de grandes choses il est agréable non seulement de passer encore pour jeune, mais d'être même dans la fleur de l'adolescence, et la réflexion sur l'âge où l'on se voit fait qu'on prête l'oreille aux conseils d'un homme usé par les travaux. Il oppose ses années à la Jeunesse de Scipion, les vicissitudes de sa fortune ${ }^{47}$, à sa prospérité constante, et pour s'emparer plus aisément de son cœur il ajoute que ces réflexions l'alarment sur le sort et sur les intérêts de Scipion.

\section{[Cb]}

Les Maximes générales et qui ont l'évidence des Notions communes sont d'une grande efficace pour persuader : Hannibal s'en sert et dit,

NON TEMERE INCERTA CASUUM REPUTAT QUEM FORTUNA NUNQUAM DECEPIT.

JE NE M'ÉTONNE PAS QU'ON NÉGLIGE DE RÉFLÉCHIR SUR L'INCERTITUDE DES ÉVÉ NEMENTS, QUAND ON N'A ENCORE JAMAIS ÉTÉ TROMPÉ PAR LA FORTUNE. 
Il excuse Scipion, Il n'a pas encore passé par les circonstances qui rendent les hommes prudents et auxquelles Hannibal lui-même [44] reconnaît qu'il doit ce qu'il se trouve de sagesse et de modération.

\section{[Cc]}

Pour s'emparer toujours plus de l'attention et du cœur de Scipion, il lui met en peu de mots devant les yeux l'histoire de sa propre Vie, et la lui peint dans tout son beau ${ }^{48}$.

[§12] Quod ego fui ad Trasimenum, ad Cannas, id tu hodie es, vix dum militari aetate imperio accepto, omnia audacissime incipientem nusquam fefellit fortuna: [\$ 13] Patris et Patrui persecutus mortem, ex calamitate uestrae domus decus insigne uirtutis pietatisque eximiae cepisti: Amissas Hispanias recuperasti, quatuor inde Punicis exercitibus pulsis; [§ 14] Consul creatus, cum caeteris ad tutandam Italiam parum animi esset, transgressus in Africam, duobus hic exercitibus caesis, binis eadem hora captis simul incensisque castris, Syphace potentissimo Rege capto, tot urbibus Regni eius, tot nostri Imperii ereptis, me sextum decimum iam annum haerentem in possessione Italiae detraxisti.

Ce que j'ai été à Trasimène et à Cannes vous l'êtes aujourd'hui. Vous vous êtes vu à la tête des armées dans un âge où à peine on commence à porter les armes, et quelques hardis qu'aient été vos projets, la Fortune les a toujours parfaitement secondés. Armé pour venger la mort de votre Père et de [45] votre Oncle, les malheurs de votre famille ont servi à relever la gloire ${ }^{49}$ de votre Valeur et de votre Piété ; Vos troupes victorieuses, ont remis les Espagnes, que l'on comptait perdues pour jamais, sous le pouvoir des Romains ${ }^{50}$. Vous avez forcé quatre armées ennemies à les abandonner. Élevé ensuite au Consulat dans le temps que le reste des Romains avaient à peine assez de courage pour se promettre de conserver ${ }^{51}$ l'Italie, vous passez en Afrique, vous y taillez en pièces deux armées; d'un seul jour vous vous rendez le Maître de deux Camps; vous y portez et le fer et le feu; Syphax le plus puissant de nos Rois devient un de vos prisonniers. Vous vous rendez enfin Maître de tant de places vous en enlevez un si grand nombre à Syphax et aux Carthaginois, que vous m'arrachez ${ }^{52}$ à l'Italie dans la possession de laquelle je me comptais affermi depuis seize années.

D'un côté ; quand on n'aime pas les gens, l'esprit se détourne de la vue de leur gloire ${ }^{53}$. D'un autre; si Hannibal s'était appliqué à embellir les faits de Scipion, son éloquence aurait été suspecte ; Il s'exprime donc en peu de mots, cela est plus naturel ; mais toutes ces expressions ont une force singulière. C'est ainsi que le cœur parle, ce qu'il sent avec admiration, il l'énonce aussi d'une manière propre à l'exciter ${ }^{54}$.

[46] D'abord Hannibal reconnaît dans Scipion tout ce qu'il y a de plus grand dans sa propre fortune. Quod ego fui ad Trasimenum, ad Cannas, id tu hodie es. Tout ce que j'ai été à Trasimène, et à Cannes, vous l'êtes aujourd'hui. Général à un âge où à peine on a assez de force pour être Soldat, les événements ont toujours secondé votre courage. Il ne se contente pas de le dire en gros, il se fait un plaisir de le répéter en détail ${ }^{55}$. Patris et Patrui persecutus mortem, ex calamitate uestrae domus decus insigne uirtutis pietatisque eximiae cepisti. Armé pour venger la mort de votre Père et de votre Oncle, les malheurs de votre famille ont servi à relever la gloire de votre Valeur et de votre Piété. Ce que présentait de triste la défaite de son Père et de son Oncle, il l'efface par les termes de decus; decus insigne, decus virtutis, pietatis, pietatis eximiae. Gloire, gloire des plus éclatantes, Valeur, Piété, Piété exquise.

41 Vainqueur de l'Espagne, dont le sort était décidé et qui était déjà perdue pour les Romains; amissas recuperasti et comment? Exercitibus pulsis, exercitibus quatuor. Vous en avez chassé les armées de vos ennemis. Vous avez défait quatre armées. Le discours va en croissant. Caeteris parum animi ad tutandam Italiam, transgressus in [47] Africam. À peine le 
reste des Romains osaient espérer de pouvoir sauver l'Italie, vous sûr de sa conservation, vous passez en Afrique, c'était déjà beaucoup, mais qu'y fait-il ? Il se rend maitre de deux camps, et comment? À la même heure: Il met dans les fers un Roi; un Roi très puissant : Les Villes de l'un et de l'autre Empire, tombent sous ses coups.

D'abord Hannibal avait reconnu en Scipion son égal, maintenant il le trouve au-dessus de lui ${ }^{56}$, me sextumdecimum annum haerentem in possessione Italia detraxisti, et detraxisti haerentem, haerentem in possessione, eoquè annum decimum sextum. Vous m'en avez arraché; Tout affermi que je me crusse dans sa possession, depuis l'espace de seize ans.

\section{[Cd]}

L'ambition la plus démesurée doit être satisfaite après un tel dénombrement, et quand Hannibal demande à Scipion :

[§ 15] Potest victoriam malle, quam pacem animus?

Se peut-il que votre courage soit encore plus avide d'une nouvelle victoire, que de

l'honneur d'avoir donné la Paix à Rome et à Carthage,

il a déjà préparé son cœur à répondre par ce qu'il vient de lui représenter. C'est le plus grand secret de l'Art que d'amener ainsi peu à peu son Auditeur aux Sentiments dont on veut qu'il soit pénétrés7.

\section{[Ce]}

Il n'était pas possible que le cœur de [48] Scipion ne fut ébranlé, et c'est sur les cœurs une fois ébranlés qu'il faut porter les coups les plus vifs et les plus redoublés.

Il semble qu'Hannibal lit dans le cœur de Scipion; Il y voit une grande répugnance à se rendre ; cette répugnance il ne la condamne pas, loin de cela il la regarde comme l'effet de son grand courage : Après cette insinuation, il lui est bien permis d'ajouter qu'il en craint les suites pour Scipion même. Novi uobis spiritus magnos magis quam utiles. Je vois dans vos sentiments plus de grandeur que de sûreté, et comment sait-il qu'il y a quelquefois dans le courage plus de grandeur que de prudence? Il le sait bien, car il le sait par sa propre expérience, et mihi talis aliquando fortuna affulsit. Un temps a été que ma fortune n'était pas moins brillante. Jusqu'ici Scipion est supérieur à Hannibal, mais il lui sera supérieur, en tout sens, s'il sait mieux profiter que lui de sa prospérité, et des effets de son courage ${ }^{58}$.

\section{[Cf]}

Les avis continués peuvent déplaire; Les instructions peuvent jeter dans l'impatience. Hannibal prévient ${ }^{59}$ ces mouvements en réveillant la piété de Scipion :

[\$16] QUOD SI IN SECUNDIS REBUS BONAM QUOQUE [49] MENTEM DARENT DII;

SI À PROPORTION DE LA PROSPÉRITÉ QUE LES DIEUX NOUS ENVOIENT, ILS NOUS ACCORDAIENT EN MÊME TEMPS UN ESPRIT QUI SÛT EN FAIRE USAGE ${ }^{60}$.

C'est donc un présent des Dieux de savoir penser à l'avenir, et de savoir le craindre.

Après qu'Hannibal a fait convenir Scipion que savoir profiter du passé pour prévoir et régler prudemment l'avenir, est une des plus précieuses faveurs qu'on reçoive du Ciel ; quand il l'oblige de faire attention à cette vérité, il se présente à lui comme un instrument dans la main des Dieux, et il parait parler à Scipion de leur part ${ }^{61}$. 


\section{[Cg]}

Si donner des leçons et se proposer en exemple marque quelque supériorité, quand on le fait dans un état aussi humilié que celui dans lequel Hannibal prend soin de se faire voir, on ne peut être soupçonné de présomption et de parler avec trop de hauteur.

Ut omnium obliuiscaris aliorum, satis ego documenti in omnes casus sum.

Quand vous fermeriez les yeux à tous les autres exemples, il vous suffirait de les arrêter sur le mien pour vous convaincre du parti que l'on doit tirer de l'une et de l'autre fortune.

Quand vous fermeriez les yeux à tout le reste, ut omnium obliuiscaris. Il suppose que Scipion connaît une infinité d'autres exemples, et en lui permettant de les oublier il ne laisse pas de les rappeler, au moins confusément dans sa mémoire.

\section{[Ch]}

Il a raison de dire que son exemple peut suffire : Il ne parle de sa fortune précédente que pour faire plus vivement sentir, par ses antithèses, son malheur présent ${ }^{62}:$ Il l'expose d'une manière à charmer le grand cœur de Scipion même, à la vue des vicissitudes auxquelles le Genre humain est assujetti.

[§ 17] Quem modo castris inter Anienem atque urbem uestram positis, signa inferentem ac iam prope scandentem moenia Romana uideras, hic cernis duobus fortissimis uiris fratibus clarissimis Imperatoribus orbatum ante moenia prope obsessae Patriae, quibus terrui uestram urbem, ea pro mea deprecantem.

Moi que vous avez vu, mon Camp placé entre la Rivière et la Ville, prêt de donner l'assaut à vos murailles, vous me voyez ici, privé de mes deux vaillants frères, Généraux d'un mérite éclatant, et avoué de tout le monde, vous ${ }^{63}$ supplier d'épargner aux Carthaginois ce que j'ai fait craindre aux Romains.

Videras, Cernis, prope scandentem moenia Romana ; Ante moenia obsessae Patriae; Quibus [51] terrui ea deprecantem. Vous avez vu, Vous voyez, Presque aux pieds de vos remparts et prêt de les escalader, Je vous vois presque toucher aux murs de ma Patrie assiégée. Craignant pour nous ce que j'ai fait redouter aux autres. Cernis. Vous le voyez, il le force d'y faire attention: Ces objets sont trop grands pour la leur refuser.

\section{[Ci]}

Au cœur de Scipion ébranlé par un si grand exemple, il offre une maxime générale et par là respectable. Ces Maximes sensées sont les preuves de sa tranquillitée ${ }^{64}$,et par là il donne un double poids à son discours.

[\$ 18] MAXIMAE CUIQUE FORTUNAE MINIME CREDENDUM EST.

C'EST À LA PLUS GRANDE FORTUNE QU'IL FAUT LE MOINS SE FIER ${ }^{65}$.

De la Maxime générale il passe à l'application ${ }^{66}$. Votre Fortune est très grande.

Es in rebus bonis; nos in dubiis, tibi pax ampla ac speciosa, nobis necessaria magis quam honesta.

Votre sort est heureux, le nôtre est chancelant. La paix s'offre à vous pleine de gloire et d'éclat. Pour ce qui est de nous elle ne nous fait pas honneur, mais elle nous est nécessaire.

52 Pour gagner la confiance de Scipion, il lui parle comme à un Confident à qui il ne dissimule quoi que ce soit ${ }^{67}$. 


\section{[Cj]}

\section{brillante qu'elle soit, mais plutôt s'en défier, il ajoute immédiatement.}

[§ 19] Melior tutiorque est certa pax, quam sperata uictoria, haec in tua, illa in Deorum manu est. Ne tot annorum felicitatem in unius horae dederis discrimen.

Une Paix que l'on tient déjà est de beaucoup plus estimable et plus sûre qu'une Victoire qu'on espère ${ }^{6}$. La Paix est en votre Puissance. La Victoire dépend des Dieux. Ne vous exposez pas au risque de perdre dans une heure, tout le bonheur dont vous jouissez depuis tant d'années.

Il lui met devant les yeux trois considérations, dont les dernières enchérissent sur les précédentes. Certa pax. Dubia victoria. In tuam manu, In Deorum manibus. Felicitas tot annorum ; Discriminem unius horae. La Paix est sûre. La Victoire douteuse. L'une est dans vos mains. L'autre dans celle des Dieux. Une félicité qui dure depuis tant d'années. Le danger de la perdre dans l'espace d'une heure, et encore cette félicité il l'a reçue, mais pour le danger il en sera l'unique cause.

\section{[Ck]}

C'est ce danger sur lequel il était important d'insister ${ }^{69}$ : Un grand courage s'accoutume à ne le voir pas, voilà pourquoi Hannibal s'applique à le mettre sous les [53] yeux de Scipion.

[§20] Cum tuas uires, tum uim fortunae, Martemque belli communem propone animo; utrimque ferrum, corpora humana erunt, nusquam minus quam in bello euentus respondent.

Comparez vos forces avec celles de la Fortune, et souvenez-vous que le Dieu de la guerre distribue ses faveurs avec assez d'égalité ${ }^{70}$. On en partage également les dangers. Le fer brille d'un côté autant que de l'autre, et d'un côté comme de l'autre il ne se trouve que des hommes qui en sont facilement percés: Jamais les événements ne répondent moins à nos espérances que dans une guerre continuée ${ }^{71}$.

\section{[Cl]}

56 À ces vérités d'expérience il joint des réflexions, et pèse les avantages avec les inconvénients, en supposant toujours que la prudence veut qu'on s'en tienne au plus sûr.

[\$ 21] Non tantum ad id quod data pace iam habere potes, si proelio uincas, gloriae adieceris, quantum ademeris, si quid aduersi eueniat: Simul parta ac sperata decora, unius horae fortuna euertere potest.

Ce qu'une nouvelle bataille gagnée ajoutera à votre Gloire, n'est nullement comparable à ce qu'elle perdra de son éclat si vous éprouvez quelque revers. La fortune peut renverser dans ${ }^{72}$ une heure tout le lustre où vous êtes, et tout celui que vous vous promettez.

\section{[Cm]}

Les répétitions ne déplaisent point, quand elles [54] partent d'un cœur qui aime et qu'elles sont les expressions de sa tendresse : On la sent dans ces paroles. 
[\$ 22] Omnia in pace iungenda, tuae potestatis sunt, P. Corneli, tunc ea habenda fortuna erit quam Dii dederint.

PUBLIUS CORNELIUS, vous pouvez régler en Maître absolu tout ce qui doit servir à nous unir par une bonne paix; Mais après une bataille hasardée, il faudra se contenter de la manière dont il plaira aux Dieux d'en décider.

P. CORNELI, il lui parle comme un Père ferait à son enfant: D'un côté il lui fait voir ce qu'il peut, et il emploie le mot de potestas, qui est des plus brillants ${ }^{73}$. À l'indépendance exprimée par ce terme il oppose la dépendance, quam Dii dederint. Ce qu'il plaira aux Dieux de vous accorder. Il n'y a pas moyen d'aller contre eux ; ce sera ce qu'il leur plaira. Pacem iungere, Unir les hommes par la paix, cette idée est aimable : Ce qu'il y oppose est si effrayant, que sa tendresse ${ }^{74}$ pour Scipion ne lui permet pas seulement de le lui exprimer : Il se contente de le lui faire entrevoir, et de dire en gros. Tunc ea habenda fortuna erit quam Dii dederint. Il faudra se contenter du sort que les Dieux trouveront à propos de donner.

\section{[Cn]}

Ces malheurs possibles, qu'il ne veut pas présenter comme regardant Scipion, [55] il les lui fait pourtant voir dans l'histoire d'un autre. L'exemple est vénérable par son antiquité même. Quondam : Il est très semblable, on le sent, sans qu'Hannibal en fasse l'application.

Il n'attribue pas de la férocité à la personne d'Attilius ${ }^{75}$, ce reproche serait retombé par contrecoup sur Scipion, mais seulement de l'indulgence pour une fortune trop fière, non cohibebat efferentem se fortunam: Il se laissait aller à ce qu'une fortune trop heureuse lui donnait de confiance. Remarquez encore l'Antithèse, altius, foedius.

\section{[D. Proposition des articles de paix et anticipation d'objections]}

[Da]

60 Après qu'Hannibal s'est ainsi appliqué à persuader Scipion qu'il est de son intérêt même de consentir à la paix ; Pour marquer sa franchise, et pour paraître très éloigné de toute sorte de détour, sans ajouter quoi que ce soit d'autre, il vient à en faire la proposition $^{76}$.

61 Mais n'est-ce pas au vainqueur à en proposer les articles? Si Hannibal en use autrement, ce n'est ni par ignorance, ni par grossièreté : Il sait ce qui se doit et ce qui se fait.

[§ 24] Est quidem ejus qui dat, non qui petit, conditiones dicere pacis ;

À la vérité c'est à celui qui est en pouvoir de donner la paix, et non pas à celui qui la

demande à en proposer les conditions ;

\section{[Db]}

Mais à cette vérité qu'il reconnaît, et qu'on lui pourrait objecter, il fait deux réponses ${ }^{77}$. $1^{\text {re }}$. L'éclat où s'est vue Carthage et la gloire dont elle a joui, semble exiger en sa faveur quelque exception à la règle générale, et la rendre digne de quelque ménagement dans ses malheurs. $2^{\mathrm{e}}$. Loin de proposer des Lois au Vainqueur, toute la liberté que les 
Carthaginois se donnent se réduit à prononcer eux-mêmes leur condamnation, et à se choisir leur châtiment :

Sed forsitan non indigni sumus, qui nobismet ipsis mulctam irrogemus.

Mais peut-être ne sommes-nous pas indignes de nous juger nous-mêmes, et de prononcer sur ce que nous reconnaissons d'avoir mérité.

À conditiones il oppose mulctam, à dicere conditiones, irrogare mulctam. Aux conditions il oppose une amende, à régler les conditions, il oppose, ordonner du châtiment. Encore cette liberté, ce reste de droit, il veut bien le tenir de la grâce du Vainqueur, forsitan non indigni, non indigni pour digni, forsitan pour certe. PEUT-ÊTRE ne sommes-nous pas INDIGNES. Non indignes pour Dignes, Peut-être, pour certainement ${ }^{78}$.

\section{[Dc]}

[§ 25] Non recusamus, quin omnia, propter quae ad bellum initum est, uestra sint, Sicilia, Sardinia, Hispania, quidquid Insularum toto inter Africam, Italiamque continetur mari.

Nous ne faisons aucune difficulté de vous abandonner tout ce qui a été l'occasion de [57] cette Guerre. La Sicile, la Sardaigne, l'Espagne ; Tout ce que la mer environne d'îles entre l'Italie et l'Afrique.

Mais que nous donnez-vous? Tout ce dont vous êtes en possession, nous ne nous opposons plus à ce que vous le regardiez comme vôtre en toute propriété; nous consentons à vous en reconnaître les légitimes Souverains : Nous ne nous réservons que notre Ville et son territoire, pour voir de dessus nos murs votre vaste Empire. Hannibal veut bien que Scipion s'aperçoive que ni lui, ni ses Citoyens ne sont point insensibles à la grandeur de leur perte, il la propose d'une manière à exciter même la pitié du vainqueur.

\section{[Dd]}

[§ 26] Carthaginienses inclusi Africae litoribus, uos quando ita Diis placuit, externa etiam terra marique uideamus regentes imperia.

Les Carthaginois se renfermeront dans les bords de l'Afrique. Mais pour vous puisque les Dieux l'ont ainsi trouvé bon, nous vous verrons et sur Terre et sur Mer en possession des Royaumes, qui avaient jusqu'ici reconnu d'autres Souverains.

63 Quando ita Diis placuit, il se résigne à la volonté des Dieux ; Il n'est plus permis d'insulter à ceux qui s'y soumettent. La pratique d'un si grand devoir les rend trop vénérables.

D'ailleurs puisqu'ils regardent leur [58] abaissement et la paix où il les réduit, comme un effet de la volonté des Dieux, ils sentent l'obligation où ils sont d'y acquiescer, et de garder cette paix qu'ils ont eux-mêmes demandée, et dont ils ont prononcé les articles.

\section{[De]}

Il s'ouvre donc déjà par ces paroles ${ }^{79}$, une voie pour résoudre une objection très forte, et qu'il prévoit qu'il va lui-même proposer; Elle se présentait trop naturellement pour la dissimuler.

[§ 27] Haud negauerim, propter non nimis sincere petitam, aut expectatam nuper pacem, suspectam esse uobis Punicam fidem.

Je ne disconviendrai pas que vous pouvez nous accuser de n'avoir pas demandé la paix avec assez de sincérité, il y a quelque temps, ou de n'avoir pas attendu votre réponse avec assez de bonne foi, et je comprends que le souvenir de cette faute peut encore nous rendre suspects aujourd'hui. 
Proposer une objection dans toute sa force c'est une preuve de la confiance que l'on a dans la réponse qu'on lui prépare, et par laquelle on va la dissiper ${ }^{80}$. Il ne se récrie point sur le Proverbe de Punica fides, la bonne foi des Carthaginois, comme sur une dureté. Il reconnaît qu'ils ont mérité ce reproche tout récemment même, propter non nimis sincere petitam aut expectatam [59] nuper fidem. Pour n'avoir pas demandé la paix avec sincérité, pour ne l'avoir pas attendue avec assez de bonne foi.

Multum per quos petita sit, ad fidem tuendae pacis pertinet Scipio : [\$ 28] Vestri quoque, ut audio, patres nonnihil etiam ob hoc, quia parum dignitatis in legatione erat negauerunt pacem. [\$ 29] Hannibal peto pacem, qui neque peterem, nisi utilem crederem; et propter eandem utilitatem, tuebor eam, propter quam petii. [§30] Et quemadmodum quia a me bellum coeptum est, ne quem eius poeniteret, quoad ipsi inuidere Dei, praestiti, ita annitar, ne quem pacis per me partae poeniteat.

Mais Scipion faites attention, $\grave{a}^{81}$ l'influence que le caractère de ceux qui demandent la paix doit naturellement avoir sur sa durée. Vos Sénateurs eux-mêmes, à ce que l'on m'a assuré, se déterminèrent à nous la refuser ${ }^{82}$, parce qu'ils ne trouvaient pas assez de dignité, dans les Ambassadeurs qui leur en faisaient la proposition ${ }^{83}$. Mais aujourd'hui c'est Hannibal lui-même qui demande la Paix, qui ne la demanderait pas, s'il n'était convaincu de son utilité ; et qui aura soin de la rendre constante, en vue des mêmes intérêts qui la lui font souhaiter. Parce que j'ai été le premier Auteur de cette guerre ; j'ai pu faire, pendant que les Dieux ne m'ont pas regardé de mauvais œil, que personne n'eut [60] sujet de s'en plaindre. J'en userai de même, et je donnerai toute ${ }^{84}$ mon application à faire que qui que ce soit n'ait regret à une paix que j'aurai procurée.

Il reconnaît les raisons que l'on a de se défier de ses concitoyens; il ne cherche point à insinuer que les Carthaginois vont changer d'humeur et devenir des gens de bonne foi. Cette promesse aurait été trop hardie et trop peu croyable. Il consent qu'on les regarde comme des gens, sur qui l'intérêt seul peut beaucoup et l'honneur quelque peu ${ }^{85}$.

Multum per quos petita sit, ad fidem tuendae pacis pertinet, Scipio. Scipion, nous conférons ensemble, parlons à cœur ouvert. Tout homme qui a travaillé à procurer une paix est intéressé à la faire regarder comme avantageuse, et s'il est puissant dans sa République, il en viendra à bout. Je suis la ressource des Carthaginois pour la guerre, et vous celle des Romains. Quand nous l'aurons terminée par la paix, nous ne troublerons pas notre ouvrage, et si nous nous unissons à le maintenir, qui est-ce qui le troublera?

Vestri quoque, ut audio, patres nonnihil etiam ob hoc, quia parum dignitatis in legatione erat, negauerunt pacem. Votre Sénat l'a refusée la paix, parce que ceux qui la lui [61] proposaient, ne lui en paraissaient pas d'assez bons garants.

Hannibal peto pacem, qui neque peterem nisi utilem crederem; et propter eandem utilitatem tuebor eam propter quam petii. C'est Hannibal lui-même qui demande la paix, qui ne la demanderait pas, s'il n'était convaincu de son utilité, et qui aura soin de la rendre constante en vue des mêmes intérêts qui la lui font souhaiter. Il ne parle que de ses intérêts ${ }^{86}$, et non point de ceux de Scipion, pour lui faire connaître qu'il compte sur sa bonne foi, satisfait que Scipion de son côté le regarde comme un homme qui connaît ses intérêts, et qui est assez raisonnable pour ne leur être pas contraire.

71 Et quemadmodum, quia a me bellum coeptum est, ne quem eius poeniteret quoad ipsi inuidere Dei, praestiti; ita annitar ne quem pacis per me partae poeniteat. Parce que j'ai été le premier Auteur de cette Guerre, j'ai pu faire, pendant que les Dieux ne m'ont pas regardé de mauvais œil, que personne n'eut sujet de s'en plaindre. J'en userai de même, et je donnerai toute mon application à faire que qui que ce soit n'ait regret à une paix que j'aurai procurée. J'ai conseillé 
la guerre, et que n'ai-je point fait pour la soutenir ? Quand j'aurai conseillé la paix, il me sera infiniment plus aisé de l'affermir.

[62] Le peuple de Carthage souffrira infiniment moins de la paix qu'il n'a souffert de la guerre. Et que n'ai-je point été obligé de faire pour détourner son attention de ses pertes et pour adoucir le sentiment de ses dépenses. Mes succès m'élevaient au-dessus de l'homme, et égalaient en quelque sorte ma gloire à celle des Immortels; elle leur a paru trop grande ; Ils l'ont vue de mauvais œil. Invidere Dei. Ils m'ont traité comme si j'avais excité leur jalousie ${ }^{87}$. Ce dernier trait allait à engager Scipion à des réflexions les plus sérieuses sur la félicité humaine, dont pour l'ordinaire la plus brillante est celle qui dure le moins ${ }^{88}$.

\section{NOTES}

1. Rendre les auditeurs attentifs (attention) et bienveillants (cœur): Aristote, III, 14, à propos de l'exorde, mais en précisant que cela doit se faire tout au long du discours. S'emparer : c'est la captatio. Même mot plus bas p. 43 et 44 . On trouve aussi : gagner (la confiance, p. 28, 31, 51; le cœur, p. 29 ; une âme, p. 42 ; une personne, p. 28, 41).

2. Soutenir/démentir son caractère: terme de critique classique pour parler d'un des quatre principes aristotéliciens (Poétique, 54a 16) de construction du personnage, la «constance » ou " égalité de mœurs ".

3. Faire connaître, faire sentir : renvoi à l'idée des sèmeia et des erga de la vertu, ses signes et les effets manifestes (Aristote, I, 9, 1366b 25-35), analogues aux signes dans la démonstration (I, 2, 1357 b1 sq.).

4. Grandeur de ses sentiments : magnanimité, megalopsukhia, Aristote, I, 9, 1366 b 1 sq. Une des neuf vertus aristotéliciennes.

5. Compassion : eleos, pitié (Aristote, II, 8), une des passions.

6. Ici commence l'analyse que fait Hannibal de la situation («il se voit », "il juge », etc.) en homme prudent qu'il est (prudent au sens de la prudentia). Mais le passage est aussi un exposé de ce qui permet de «bien juger» le discours, sujet, circonstances, caractères: voir première phrase.

7. C'est l'ingenium qui permet l'invention. Le mot est à distinguer de l'«esprit» et de l'« expérience ",judicium et usus, qui ne permettaient que l'analyse de la situation.

8. Quelle vertu désigne exactement cette «politesse»? Les trois vertus sont de nouveau associées plus loin (p. 31) : «il ne se pouvait que Scipion, dont l'Âme était véritablement grande, et qui avait une grande piété et une grande politesse, ne fût sensible à ces douceurs, et ne souhaitât de mériter toute cette estime et toute cette affection, dont Hannibal lui donnait de si fortes assurances. » Ce passage semble associer la politesse à la passion de l'amitié, philia, suscitée par le geste de bienveillance, Aristote, II, 4, 1381 b 35. Voir, p. 34 : «Un cœur ambitieux et généreux refuse difficilement son affection à celui qui fournit la plus grande matière de sa gloire ». Le début du commentaire de la réponse de Scipion l'associe plutôt à la passion de la reconnaissance, kharis (Aristote, II, 7) : «Scipion, pour ne commettre point sa politesse, et ne s'exposer point au risque de paraître insensible », "Quelle obligation vous en ai-je... » Peut-être généralement le fait de paraitre bon (spoudaios, Aristote, II, 1, 1378 a). C'est surtout une vertu qui permet de jouer sur la passion de la honte, souffrance liée à l'idée d'une perte de réputation 
(aidôs, Aristote, II, 6, 1383b) : "Scipion qui dès sa jeunesse s'était rendu respectable par sa pitié " (commentaire au discours d'Hannibal, p. 30). Par ses «douceurs", Hannibal met en jeu la réputation de Scipion en cas de refus. C'est ce que confirme, dans la réponse de Scipion, la traduction de «nulla sum tibi uerecundia obstrictus », par « il est visible qu'on ne saurait m'accuser de manquer à la bienséance et à la modestie, si je ne vous en marque pas ma reconnaissance». Dans le commentaire, Crousaz donne comme équivalent «modestie et politesse ». Verecundia signifie à la fois le devoir de pudeur et le sentiment de honte et est donc l'exact équivalent de l' aiskhunè et de l'aidôs d'Aristote, et il est utilisé comme tel dans les traductions anciennes. C'est par exemple verecundia qu'utilise Ferrazzi (avec celui de pudor).

9. Sur la manière dont Hannibal joue de la piété, voir commentaires de $A b, B a, C f, C j, D d$ (et le commentaire de la réponse de Scipion cité note 88).

10. Le caractère affiché par Hannibal selon Crousaz est donc celui de l'ami (celui qui veut votre bien non pour lui mais pour vous même : Aristote, II, 4). Crousaz indiquera plus loin les passages où Hannibal parle en père (« se présente à lui sous l'idée d'un Père que la tendresse fait parler ", p. 42) ou en confident («Pour gagner la confiance de Scipion, il lui parle comme à un Confident à qui il ne dissimule quoi que ce soit », p. 51), la parenté avec la camaraderie sont les situations par excellence qui entraîne de la philia (1381b 33-34). Mais Crousaz indiquera plus loin d'autres airs que prend Hannibal : « pour parler en homme que l'expérience a solidement instruit » (p. 38); « il se présente comme un homme à qui une Raison tranquille laisse voir les choses telles qu'elles sont » (p. 41). Rapprocher de Tesmar : l'ethos ou air de celui qui sait ce qu'il en est de la vie et de ses aléas («Ethos est viri fortunae inconstantiam probe expendentis»). Et de Ferrazzi : l'air d'un personnage à la sagesse supérieure («Mores Viri Sapentis»).

11. Voir sur l'exorde les notes 19 et 33. Tesmar : exorde tiré de la recommandation de sa propre résolution, fondé sur le destin, l'avantage qu'en tire la gloire de Scipion et l'étonnante vicissitude du sort. Ferrazzi : exorde tiré à la fois de la mauvaise fortune de l'orateur et de la gloire de l'auditeur. Lorich: exorde captant la bienveillance en se fondant sur la personne de celui qui parle («a sua persona »), mais, à partir de laetor, tentative de capter la bienveillance par la personne de Scipion (« a persona Scipionis »).

12. Voir plus bas p. $37:$ «Jusqu'ici la personne même de Scipion est entrée dans tout ce qu'Hannibal vient de dire ».

13. Var. trad. initiale, p. 3 : vois réduit, je me félicite de ce que c'est à vous.

14. Var. trad. initiale : et surtout de ce qu'il est en votre.

15. Voir supplex dans les commentaires de Ferrazzi sur le site RARE: http://w3.u-grenoble3.fr/ rare/spip/spip.php?article204. Ferrazzi ne note pas cette attitude pour Hannibal. C'est l'attitude attendue dans une demande (petitio), ici demande de paix. Lorich fait du discours une suasio : " Generis deliberatiui oratio, Suasionem enim habet pacis, conditionibus certis componendae.» ("Discours de genre délibératif, car il contient des propos visant à conseiller la paix, des conditions sûres devant être réglées »). Tesmar fait du discours une petitio et non une suasio. Ferrazzi parle d'entrevue de négociation de paix (« impetrato colloquio cum Scipione, ita cum eo de pace agit ») et ne qualifie de suasio que le second mouvement.

16. Les capitales marquent ici le début du texte commenté. Dans la suite, elles mettront en valeur les sententiae, les maximes, les phrases remarquables. Certaines phrases mises en capitales dans la traduction initiale ne le sont pas dans le commentaire.

17. La formule mettre sous les yeux, latin ante oculos, grec pro omnatôn, signale souvent la repraesentatio, qui relève de l'evidentia ou illustratio, "cette figure qui peint les choses dont on parle, et qui, comme dit Cicéron, les met sous les yeux » (Quintilien, IX, 2, 40), qu'on appelle aussi hypotypose, et que Quintilien définit comme « une image des choses, si bien représentée par la parole, que l'Auditeur croit plutôt la voir que l'entendre ». C'est le cas p. 39 ("Vous avez vu flotter etc. »), p. 44 (« Pour s'emparer toujours plus de l'attention et du cœur de Scipion, il lui met en peu de mots devant les yeux l'histoire de sa propre Vie, et la lui peint dans tout son beau »), 
p. 53 («Un grand courage s'accoutume à ne le [le danger] voir pas, voilà pourquoi Hannibal s'applique à le mettre sous les yeux de Scipion»). Mais est-ce le cas ici ? On trouve p. 52 : « Il lui met devant les yeux trois considérations ».

18. La réflexion semble identifier l'exorde au seul $A a$, mais la suite laisse penser qu'elle s'étend sur Aa-Ac.

19. L'exorde a pour but de "faire savoir à quoi tend le discours", mais quand la cause est difficile, il vaut « mieux s'attarder sur n'importe quoi plutôt que sur l'affaire » (Aristote, III, 14). La "marche couverte» (rapprocher de «occulte interjectione verborum », Her., I, 9) désignerait donc l'exorde indirect, l'insinuatio. Le mot insinuation semble avoir le sens moderne p. 33, mais son emploi p. 48 («Après cette insinuation, il lui est bien permis d'ajouter etc. ») montre qu'il a le sens de précaution oratoire. Ni Tesmar ni Ferrazzi ne parlent pourtant d'insinuatio; Vauchelle note « exorde insinuant. Flatterie adressée à Scipion. » Et Botidoux : « exorde insinuant, tiré de la personne de l'auditeur. »

20. Ici est donné le but du discours en même temps que son moyen passionnel dominant: susciter la crainte, phobos, Aristote, II, 5 . Voir plus loin, parmi de très nombreuses autres expressions, «trembler pour l'avenir» (p.33). Même passion pour Tesmar («metus alicujus adversitatis ») et Ferrazzi ( Timor»).

21. Suggestion de la division du discours : exorde, contentio.

22. Démonstration : sèmeion du caractère (grandeur d'âme) et de l'émotion (amicitia).

23. L'opposition couvre donc une comparaison du même au même (a simili). Lorich note que les mots «si souvent, sur le point de la terminer par mes victoires » sont une allusion («significatio $»)$, ont un sens profond ; Hannibal propose son exemple pour mettre en garde Scipion : "Toties propè in manibus uictoriam habui etc. Significatio. Sensus enim profundior, quàm uerba uidentur. Quippe exemplo suo (quod ueluti robustissimum huius orationis argumentum) admonet Scipionem... » Il s'agit précisément d'une significatio per comparationem, une allusion par citation d'un cas semblable: Her., IV, 67.

24. Une des façons de faire tomber la colère et de susciter le calme : Aristote, II, 3, 1380b 14-16. Voir Ac : « Hannibal fait connaître à Scipion qu'il est déjà vengé ».

25. Adoucissement : euphémisme, deminutio dans Her., IV, 50, ou encore attenuatio. Même sens d' adoucir un peu plus loin à la même page. Avec le même sens : tempérer p. 34, effacer p. 46. La figure de style permet de rendre plus petit ce qui est considérable : c'est le contraire de l'amplification (auxesis). Voir aussi ménagement, utilisation d'«expressions les plus faibles» pour un point "délicat », p. 35, où la litote est précaution oratoire.

26. Simple/pompeuse : variation de genus dicendi, style simple et style élevé (voir " élève le style »).

27. Apaisement de la colère : voir plus haut p. 32 et la note 24 .

28. Reprise de Aa.

29. Nous rectifions l'erreur de pagination (34 et 35 pour 36 et 37).

30. Dès le début, Crousaz a donné le «principal but » d'Hannibal : «amener Scipion à réfléchir bien sérieusement sur les bizarreries du sort, et sur l'incertitude de la destinée » pour en obtenir "aisément la préférence d'une paix sûre à une victoire douteuse» (p. 31). Le commentaire par Crousaz du discours de Scipion suggère cependant deux temps (p. 67) : « Scipion a commencé par répondre à la fin du discours d'Hannibal : Il le parcourt en remontant, mais il ne touche qu'à ce qu'il a d'essentiel. Les oppositions de l'équité, des douceurs de la paix, et de sa sûreté, aux malheurs, et aux incertitudes de la guerre, est ce qu'on y trouve de plus fort, et cette opposition est énoncée d'une manière sensée, sentencieuse et touchante, dans ces paroles : Optimum quidem [...] contenti essemus. » Le premier temps ou « essentiel» du discours d'Hannibal commencerait donc avec le $\$ 6$, que nous avons ici. Le deuxième temps serait ensuite « ce que le discours d'Hannibal avait renfermé de personnel » (p. 71), à quoi Scipion «cesse de faire attention » pour «mettre sa modestie et sa politesse à couvert ». Cet aspect personnel serait donc la deuxième partie du discours d'Hannibal, dont le début de la conclusion serait un résumé: "Après 
qu'Hannibal s'est ainsi appliqué à persuader Scipion qu'il est de son intérêt même de consentir à la paix ». L'articulation semble donc se faire entre les $\S 10$ et 11 (notre $C$, p. 42 de Crousaz) : voir plus bas.

31. Var. trad. initiale : rien n'aurait pu arriver.

32. Var. trad. initiale : de tout qu'ils [sic] vous a coûté pour les acquérir.

33. Le propos laisse penser que vient de finir un exorde ab auditoris persona : « Nous obtiendrons la bienveillance des auditeurs en parlant d'eux si nous rappelons leur courage, leur sagesse, leur clémence, leur hauteur de vues dans des affaires déjà jugées; si nous montrons quelle estime on leur porte et quel jugement on attend» (Her., I, 8). Même analyse chez Botidoux. Semble aussi annoncé le début du corps du discours, puisqu'il a été dit plus haut (p. 31) qu'Hannibal tentait d'obtenir «la préférence d'une paix sûre à une victoire douteuse » en faisant réfléchir Scipion «bien sérieusement sur les bizarreries du sort, et sur l'incertitude de la destinée ».

34. Prolepse argumentative, c'est-à-dire réponse anticipée à une objection possible. Voir plus bas : «cette vérité qu'il reconnaît, et qu'on lui pourrait objecter» (p. 55, Db); « résoudre une objection très forte, et qu'il prévoit qu'il va lui-même proposer » (p. 58, De). On trouve plus loin des fragments d'une théorie de la pratique de la prolepse: aborder franchement l'objection possible («Elle se présentait trop naturellement pour la dissimuler», p. 58, De) ou au contraire la «prévenir» sans la formuler (« Il n'avance pas l'objection pour la résoudre ensuite, car il faut éviter de faire naître des défiances; Mais il lui empêche de se présenter », p. 41, fin de Bd).

35. Var. texte initial : Sicilia, atque Sardinia

36. Var. texte initial : amissis ducibus.

37. Figure de gradation. Voir plus bas, p. 46 : «Le discours va en croissant ».

38. Dans le commentaire de la réponse de Scipion, ce passage est donné comme le point fort du discours (citation complète plus haut, n. 30) : «Les oppositions de l'équité, des douceurs de la paix, et de sa sûreté, aux malheurs, et aux incertitudes de la guerre, est ce qu'on y trouve de plus fort, et cette opposition [...]. »

39. Var. trad. initiale (capitales à partir de il) : Mais IL EST [...] REMÈDE.

40. Crousaz ne nomme pas le polyptote et le chiasme syntaxique qui fait antimétabole, la commutatio dans Her. (IV, 38), mais se rappelle peut-être que la variation est associée au plaisir (Aristote, I, 11, 1371a 25).

41. Crousaz a omis la phrase qui précédait celle-ci chez Tite-Live : agimus ii quorum et maxime interest pacem esse, et qui quodcumque egerimus ratum ciuitates nostrae habiturae sunt. Dans sa traduction (p.7): «Nous qui en conférons, trouverons notre avantage à la conclure, et nos Républiques agréeront tout ce dont nous serons convenus.»

42. La vraisemblance n'est pas ici le semblable au vrai qui fonde l'enthymème (Aristote, I, 1, 1355a) et qui relève donc du logos, elle concerne l'ethos: Hannibal raconte sa vie pour se faire passer pour l'homme du raisonnable.

43. Var. trad. initiale : à tout ce que.

44. Le mot leçon apparaît en $C a$ (associé à instructions) et en $C g$ (associé à "se proposer en exemple ») et on trouve avis et instructions en $C f$. Ces mots suggèrent que cette partie du discours se donne l'allure d'une admonitio, d'une mise en garde, d'où le ton paternel. Voir site RARE, http://w3.u-grenoble3.fr/rare/spip/spip.php?article273. On passe ici de l'espérance à la crainte, deux passions du délibératif. La crainte était présente avant, mais semée parmi les flatteries et l'espérance (« espérer » en $B c$, « espérance » dite " agréable » et associée à des "agréments de paroles » en $B d$ ).

45. Var. trad. initiale : Mais, Scipion, votre.

46. Var. trad. initiale : s'est encore jamais.

47. Lorich : « a contrario ».

48. Plus haut (fin de B, p. 41), Hannibal faisait « le portrait abrégé [...] de sa vie »; ici il peint la vie de Scipion. Ce qui nous apparaît comme un récit est en effet donné par Crousaz comme une 
peinture, une hypotypose. Peinture «dans tout son beau »: il s'agit d'une amplification, d'une exagération, " dont l'objet est de relever les objets, de les faire paraître plus considérables, d'ajouter aux vertus et aux vices des qualités qui rendent les unes plus aimables et les autres plus odieuses. [...] On emploie souvent cette figure dans la composition des caractères, principalement dans le drame.» (Encyclopédie littéraire, Paris, J.P. Costard, 1772). «En peu de mots»: l'amplification n'est pas toujours développement, l'exagération est aussi synonyme d'emphase au sens rhétorique d' "expression fort significative» (de significatio). Voir plus loin (p. 45, Cc) : «si Hannibal s'était appliqué à embellir les faits de Scipion, son éloquence aurait été suspecte ; Il s'exprime donc en peu de mots, cela est plus naturel; mais toutes ces expressions ont une force singulière ». Que « peindre dans tout son beau » n'est pas du tout « embellir les faits » marque la différence entre amplification et hyperbole.

49. Var. trad. initiale : Oncle, vous avez tiré des malheurs de votre famille de quoi relever la gloire.

50. Var. trad. initiale : les Espagnes sous la Puissance des Romains, dans le temps qu'on les comptait perdues pour eux.

51. Var. trad. initiale : de courage de pouvoir défendre et se conserver.

52. Var. trad. initiale : à ce Monarque et aux Carthaginois, que vous venez à bout de m'arracher.

53. Preuve d'amicitia, la passion de celui qui veut votre bien.

54. Principe classique de la passion qui ne se communique que si elle est éprouvée. Il faut être ému pour émouvoir : Horace, Art poétique, v. 102, «Si vis me flere, dolendum est / primum ipsi tibi »; Boileau, « Pour me tirer des pleurs il faut que vous pleuriez » (Art poétique, III, v. 142).

55. Le procédé est nommé plus bas (p. 47) : «dénombrement », qui est le fait de passer de la définition de la chose à l'énumération des parties. C'est ici une amplification et les «et comment? " marquent les différentes circonstances ou «adjoints ». Ferrazzi : «il énumère [recenset] les hauts faits de Scipion, et leur donne un maximum d'importance [amplificat] en rappelant la totalité des données [ab Adjunctis ubique]». Lorich: il l'invite à la paix par la comparaison et l'énumération des divers événements ( Comparatione et Enumeratione uariarum rerum foelicissime gestarum pacem suadet»). Tesmar ne note que la comparaison des deux situations («tempora comparat utriusque »). Pour l'opposition en gros/en détail, voir le totum/omnia de Quintilien sur la repraesentatio, l'hypotypose, IX, 2, 40, rendu de même en 1718, par la même formule : « lorsqu'au lieu d'indiquer simplement un fait, on veut montrer comment il s'est passé, non en gros, mais en détail » (De l'institution de l'orateur traduit par M. l'abbé Gedoyn, Paris, G. Dupuis, p. 581); voir aussi plus bas chez Crousaz $(C d$, p. 47$)$ : «il a déjà préparé son cœur à répondre par ce qu'il vient de lui représenter. » Voir ici à l'inverse p. $54(\mathrm{Cm}):$ « Il se contente de le lui faire entrevoir, et de dire en gros. "

56. Plus haut p. 46 ( «L discours va en croissant ») était notée une figure de mot, la gradation. Ici on ne peut parler de gradation que comme figure de pensée.

57. Le passage $C a-C f(\S 11$-début du $\S 16)$ est donc une préparation, une sorte d'exorde indirect de la seconde partie, la mise en garde : «préparer son cœur ». Le début du commentaire de $\mathrm{Ce}$ en annonce la fin: les expressions ébranler le cœur et coups renvoient au movere, au flectere. La division n'est pas faite selon le logos, mais le pathos. Mais ce début de Ce est encore dans la préparation: insinuation y a le sens qu'on donne après Rollin à précaution oratoire, qui le rapproche de l'insinuatio, l'exorde indirect. Plus sensible au logos, Lorich y voit une anthupophora (contre-hypophore, Quintilien, IX, 2, 106), c'est-à-dire une prolepse argumentative, la réponse anticipée à une objection possible.

58. Scipion a les vertus de la jeunesse (courage), il pourrait avoir aussi celle de l'âge mûr (prudence, ou plutôt prudentia). Voir plus bas, p. 43 : «Il excuse Scipion, Il n'a pas encore passé par les circonstances qui rendent les hommes prudents. »

59. Le verbe prévenir n'a pas ici une portée argumentative (prolepse), comme il l'avait plus haut (« Hannibal prévient cette objection», p. 47). Il s'agit bien d'anticiper, mais du point de vue du pathos, non du logos : apaiser par avance par une passion (pitié) une autre passion, ici la colère : 
voir Achille impiger, iracundus, inexorabilis chez Horace (Art poétique, v. 121) et « impatient » dans l' Iphigénie de Racine (I, 1, v. 97), calqué sur impatiens irae.

60. Crousaz accentue l'effet de maxime en ne reprenant pas la suite immédiate du texte de TiteLive : "non ea solum quae euenissent sed etiam ea quae euenire possent reputaremus». Dans sa trad., p. 11 : «nous ne compterions pas moins sur les revers qui pourraient survenir que sur les succès passés ».

61. «Savoir profiter du passé etc.» renvoie à $B b$, p. 39, à propos du $\S 8:$ «Des idées du passé Hannibal vient donc au présent, pour le mieux régler ». L'utilisation de la pitié plus haut, l'aspect récapitulatif et le fait qu'Hannibal se présente comme parlant au nom des dieux semblent suggérer que le passage est une péroraison "partielle». Il s'agit en fait de la fin de la préparation : la « leçon » commence.

62. L'opposition du malheur présent au bonheur passé est un des lieux de la pitié (le premier de la liste donné par Cicéron, De inventione, I, LV, 107).

63. Var. trad. initiale : mérite avoué, vous.

64. La tranquillité d'esprit est une passion, to tharrein, confidentia, c'est la passion opposée de la crainte (Aristote, II, 5). Crousaz utilise le mot preuve qui relève de la démonstration pour parler du caractère et de la passion de l'orateur (il reste certes en cela fidèle à l'usage polyvalent qu'Aristote fait du mot «signe », d'autant que le signe sûr, tekmèrion, est une preuve). Là encore intérêt plus grand pour le pathos que le logos : la valeur argumentative de la maxime ne sera noté qu'ensuite.

65. Texte trad. initiale : pas en capitales.

66. Application désigne ici le procédé de raisonnement faisant passer du général (la " maxime ", c'est-à-dire le grand principe) au particulier, de la thesis ou causa à l'hypothesis ou quaestio. Corneille trouve ainsi plus naturel dans le discours ordinaire la "réduction de la thèse à l'hypothèse "; il suggère donc de substituer à une formule générale comme "l'amour donne beaucoup d'inquiétude à l'esprit qu'il possède », une formule particulière comme "l'amour vous donne beaucoup d'inquiétude " (premier des Trois discours, " De l'utilité et des parties du Poème dramatique ", Trois discours sur le poème dramatique, éd. B. Louvat et M. Escola, Flammarion, 1999, p. 67). Plus bas dans le texte de Crousaz (Cn, p. 55), " application » non d'une " maxime », mais d'un exemple, c'est-à-dire raisonnement fondé sur la comparatio a simili, le rapprochement des semblables - mais le grand exemple a lui aussi une valeur générale.

67. Le discours feint de se présenter comme le conseil, qui implique la franchise.

68. Texte trad. initiale : texte en capitales.

69. Insister a ici un sens proche de amplificare: cela se fait au moyen de l'hypotypose, de la «mise sous les yeux ".

70. Var. trad. initiale : en capitales depuis « le dieu».

71. Var. trad. initiale : en capitales à partir de « jamais ».

72. Var. trad. initiale : peut vous enlever dans.

73. Le commentaire correspond à la traduction : « Maître absolu».

74. Tendresse correspond à la passion (feinte) de l'amicitia.

75. Crousaz renvoie ici de façon elliptique au $\S 23$ de Tite-Live, qui est la célèbre histoire de Régulus (Marcus Atilius Regulus). Mais il ne donne pas le texte, que voici : «[\$23] inter pauca felicitatis uirtutisque exempla M. Atilius quondam in hac eadem terra fuisset, si uictor pacem petentibus dedisset patribus nostris; sed non statuendo felicitati modum nec cohibendo efferentem se fortunam quanto altius elatus erat, eo foedius corruit. » Dans la trad. de Crousaz, p. 15 : « Autrefois Attilius s'est trouvé dans la situation où vous êtes aujourd'hui, et quel n'aurait pas été son bonheur, si couronné de la Victoire il avait consenti à donner la Paix à nos Ancêtres qui la lui demandaient ; mais pour n'avoir voulu donner aucune borne à sa félicité, et n'avoir point su résister à une Fortune qui l'élevait si haut, il tomba, et la honte de sa chute répondit à l'éclat de son élévation. » 
76. Dans le commentaire de la réponse de Scipion, Crousaz fait commencer à la proposition de paix la fin du discours ("par où Hannibal venait de finir »). Le dernier retour à la ligne dans sa traduction initiale est au début du $D$. Vauchelle et Botidoux font des conditions de paix une partie à part, mais suivie d'une péroraison. Tesmar et Ferrazzi incluent ces conditions de la paix dans la contentio ou argumentation, les considérant comme un argument de plus en faveur de la paix.

77. Ni Tesmar ni Ferrazzi n'analysent avec précision cette phrase, comme le fait Crousaz. En revanche, Lorich indique: arguments fondés sur le raisonnable, le décent et le juste ( $A$ Consentaneo, decenti, iusto pacem suadet ").

78. Adoucissement (deminutio, voir note 25) non explicité.

79. Encore un passage qui tout en ayant sa fonction propre sert de précaution oratoire.

80. La prolepse argumentative est ici moins pensée en terme de logos que de pathos: c'est l'assurance de l'orateur qui convainc plus que la raison, le dire plus que le dit.

81. Var. trad. initiale : attention, je vous prie, sur.

82. Var. trad. initiale : refusèrent d'entrer en négociation.

83. Var. trad. initiale : qui la leur proposaient.

84. Var. trad. initiale : plaindre. Je donnerai de même toute.

85. Le choix du lieu délibératif efficace (honorable/utile) signe le caractère de la nation, qui est machiavélique, adepte de la seule Realpolitik.

86. Lorich interprète les choses autrement: Hannibal revient de nouveau à sa propre personne, ici non sur sa vie mais sur son importance sociale (« rursus à suae personae dignitate pacem suadet »).

87. Dans le commentaire de la réponse de Scipion, Crousaz (p. 74) montre qu'Hannibal ici se trahit: "Hannibal essaye de l'amener à ce qu'il lui demande par des réflexions où il entre des Sentiments de piété, mais cette piété n'est pas soutenue. Il se plaint des Dieux. Ils ont presque été jaloux de son sort. Scipion pense plus juste [...]. »

88. Tesmar, Ferrazzi, Botidoux et Vauchelle voient une péroraison aux $§ 27-30$, et même une péroraison «forte» (pour Botidoux), en insistant sur la prolepse argumentative (Tesmar, Ferrazzi) ou sur la promesse (Vauchelle). Lorich se contente de noter qu'Hannibal clôt son discours sur un argument du même au même ("j'en userai de même»). Crousaz note bien l'articulation au $§ 27$ (début de la prolepse argumentative), mais ne parle pas de péroraison. Il n'emploie d'ailleurs le mot pour aucun des quatre discours. Peut-être en voit-il cependant une dans le «dernier trait » de la dernière phrase, auquel il consacre un paragraphe entier : il y a en effet reprise de l'axe annoncé au tout début, puisque « réflexions les plus sérieuses sur la félicité humaine » reprend « réfléchir sur les bizarreries du sort etc.» de Aa, p. 31 (la vraie propositio serait alors là, et non dans la « proposition » de paix). Et surtout il y a excitation d'une passion, la crainte. Crousaz joue probablement sur les deux sens de trait: détail qui fait signe, mais aussi envoi d'un projectile, le second sens reprenant l'image du coup qui ébranle le cœur en $\mathrm{Ce}$. Le dernier trait est donc le coup de grâce, la péroraison. Certes, Tesmar ( Pathos, alicujus adversitatis $»)$ et Ferrazzi ( Motus, Timor») font eux aussi de la crainte la passion dominante. Mais, commentant la fin du discours, Crousaz essaie de rendre plus sensible à la manipulation psychologique jouant sur la crainte (que devra avoir Scipion lui-même dans le cas d'un refus de la paix) qu'à l'argumentation proprement dite (visant au contraire à rassurer les Romains en cas d'acceptation de la paix). C'est à l'inverse dans l'exorde que Ferrazzi insiste sur l'excitation de la crainte, à laquelle il joint l'espoir, les deux passions du délibératif : «Il évoque discrètement [attingit] dans l'exorde ses propres revers de fortune et en même temps mentionne la gloire qu'allait obtenir Scipion d'un arrêt de la guerre et d'une paix demandée par Hannibal ; ainsi, en partie par la crainte [timor] d'une issue finale incertaine, en partie par l'espoir [spes] d'une gloire certaine, il incline l'âme de son adversaire [consilium flectat] au choix de faire la paix. » 


\section{AUTEURS}

JEAN-PIERRE DE CROUSAZ

1663-1750 